\title{
Beta dispersion and portfolio returns
}

\author{
Kyre Dane Lahtinen ${ }^{1} \cdot$ Chris M. Lawrey $^{1} \cdot$ Kenneth J. Hunsader $^{1}$
}

Published online: 6 December 2017

(C) Macmillan Publishers Ltd., part of Springer Nature 2017

\begin{abstract}
As any well-versed investor should know, there are many ways in which beta can be calculated based on factors such as the choice of time interval and market proxy used in the estimation process. Of course, this can lead to wide variation in beta estimates reported through publication sources. In this paper, we create portfolios based on the dispersion in the estimate of 27 different beta calculations. Defining stocks with higher variation in their beta estimates as higher risk, and consistent with risk-return theory, we find that portfolios of stocks with high dispersion across beta estimates outperform portfolios of stocks with low dispersion regardless of their level of systematic risk.
\end{abstract}

Keywords Beta $\cdot$ Volatility $\cdot$ Risk and return

JEL Classification $\mathrm{G} 10 \cdot \mathrm{G} 11 \cdot \mathrm{G} 12$

\section{Introduction}

Beta measures the risk that an asset contributes to a welldiversified portfolio, which is considered the relevant risk of an asset by academics and practitioners. It has become a

Chris M. Lawrey

clawrey@southalabama.edu

Kyre Dane Lahtinen

klahtinen@southalabama.edu

Kenneth J. Hunsader

khunsader@southalabama.edu

1 Department of Economics and Finance, Mitchell College of Business, University of South Alabama, Mobile, AL 36688, USA standard metric reported by most major sources of financial news such as Yahoo Finance, Google Finance, Morningstar, Barron's, the Wall Street Journal, Nasdaq.com, and dozens of other reputable sources of financial data. However, on any given day the betas reported on these sites may vary widely, which could lead to substantial confusion by potential investors. For example, on March 14, 2017, Yahoo Finance, Google Finance, and Nasdaq.com reported Apple Inc.'s beta to be $1.45,1.25$, and 0.72 , respectively. Apple's stock is one of the most popular and heavily traded stocks in the world with average daily volume over 28 million shares, yet its publicly reported betas differ drastically. Yahoo's beta estimate is more than double the beta reported from Nasdaq.com. More importantly, these extremely different betas could lead to dramatically different conclusions about the riskiness of Apple's stock. A beta of 1.45 indicates that Apple's stock is considerably riskier than the market or average stock, while the beta obtained from Nasdaq.com indicates that Apple is a lowrisk stock that has significantly less risk than the overall market or average stock. Our estimates of Apple's beta on August 9, 2010, yield dramatically different estimates from a low of 0.744 based on daily returns regressed on the Center for Research in Security Prices (CRSP) valueweighted index to a high of 1.1526 based on monthly returns regressed on the S\&P 500 index. See Table 1 and Fig. 1 for all 27 estimates for Apple's beta on August 9, 2010.

Another example of a popular and heavily traded stock with significantly different reported betas is Wal-Mart, Inc. Yahoo Finance reports its beta at 0.03 , while Nasdaq.com reports a beta that is over eight times greater at 0.25 , which again could lead potential investors to much different conclusion about how much risk that Wal-Mart stock would contribute to their portfolios. The primary reason for 
Table 1 Example of beta estimates for Apple, Inc.

\begin{tabular}{lllll}
\hline High & Low & Mean & Median & SD \\
\hline Panel A & & & & \\
1.152615 & 0.7443098 & 1.000536304 & 1.018075 & 0.113892061 \\
\hline Model & Return & Market & Observations & Beta \\
$\#$ & window & proxy & & \\
\hline Panel $B$ & & & & \\
1 & Daily & CRSP Equal & 63 & 0.7808875 \\
2 & Daily & S\&P 500 & 63 & 0.7618102 \\
3 & Daily & CRSP Value & 63 & 0.7443098 \\
4 & Daily & CRSP Equal & 126 & 1.0248 \\
5 & Daily & S\&P 500 & 126 & 0.9790519 \\
6 & Daily & CRSP Value & 126 & 0.9578621 \\
7 & Daily & CRSP Equal & 252 & 1.035572 \\
8 & Daily & S\&P 500 & 252 & 1.042041 \\
9 & Daily & CRSP Value & 252 & 1.005463 \\
10 & Weekly & CRSP Equal & 52 & 0.8913658 \\
11 & Weekly & S\&P 500 & 52 & 0.9319599 \\
12 & Weekly & CRSP Value & 52 & 0.9069445 \\
13 & Weekly & CRSP Equal & 104 & 0.9530234 \\
14 & Weekly & S\&P 500 & 104 & 1.127908 \\
15 & Weekly & CRSP Value & 104 & 1.074223 \\
16 & Weekly & CRSP Equal & 156 & 0.9328029 \\
17 & Weekly & S\&P 500 & 156 & 1.085708 \\
18 & Weekly & CRSP Value & 156 & 1.052272 \\
19 & Monthly & CRSP Equal & 36 & 0.9632296 \\
20 & Monthly & S\&P 500 & 36 & 1.105754 \\
21 & Monthly & CRSP Value & 36 & 1.093181 \\
22 & Monthly & CRSP Equal & 48 & 0.9968866 \\
23 & Monthly & S\&P 500 & 48 & 1.134247 \\
24 & Monthly & CRSP Value & 48 & 1.121986 \\
& Monthly & CRSP Equal & 60 & 1.018075 \\
\hline & Monthly & S\&P 500 & 60 & 1.152615 \\
25 & Monthly & CRSP Value & 60 & 1.140501
\end{tabular}

This table contains the example output for Apple, Inc., ticker "AAPL," for August 9, 2010. Panel A contains the summary statistics of the beta calculations for that day. Panel B contains the actual individual beta calculations for each of our 27 different models. The output from panel $\mathrm{A}$ is used in our portfolio formation step

these dramatically different beta estimates is due to the widely varying methods for estimating betas. Statistically, beta is a regression coefficient that is estimated by regressing a stock's returns against corresponding market returns. It is not a complicated operation and can easily be estimated by any spreadsheet or statistical package. The dramatic differences depend upon choice of time intervals: daily, weekly, monthly, or even annual returns; and the choice of market proxy: S\&P 500, CRSP equal weighted, CRSP value weighted, Wilshire 5000, or the Dow Jones

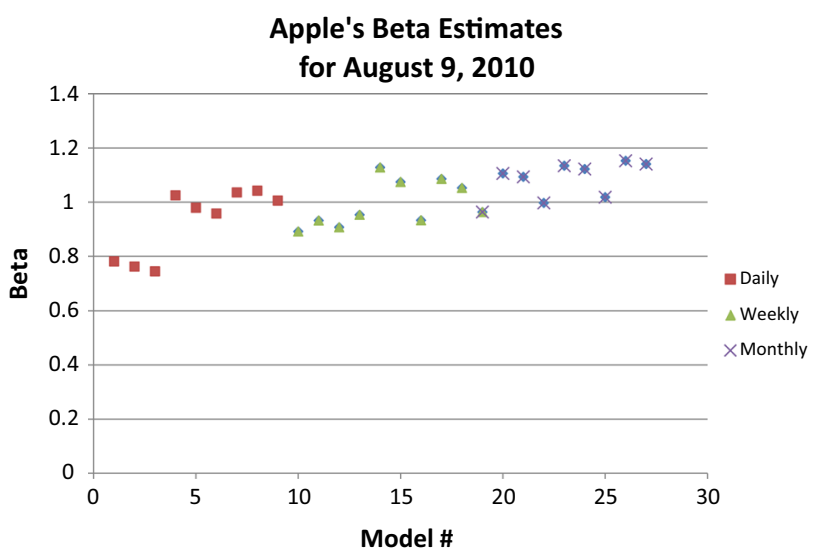

Fig. 1 Apple's beta estimates

Industrial Average (DJIA). In this paper, we extend the existing literature by analyzing the empirical relation between risk and return through a new measure of volatility using different beta calculation methods. More specifically, we calculate beta dispersion, the standard deviation of the 27 different estimates of beta, calculated with varying time intervals and market proxies. From these estimates, at the end of each month (or year), we construct portfolios based on the dispersion measure and level of beta to examine different portfolio returns.

The results show statistically significant return differences between low-beta dispersion portfolios and high-beta dispersion portfolios with the largest returns in the lowest beta quintile, indicating that low-beta estimates may not be fully capturing all of the "true or relevant" risk of those securities.

The remainder of the paper is organized as follows: "Literature review" section is a literature review. "Methodology and data" section explains the methodology and the data. "Results" section presents the estimation results, and "Conclusion" section concludes.

\section{Literature review}

Optimal beta calculations have been a recurring issue since the concept of beta was initially introduced by Sharpe (1963). Brenner and Smidt (1977) argue that accurate beta estimates are important for understanding risk-return relations and in making investment decisions. Baesel (1974) suggests that the stability of beta is dependent on the estimation interval and suggests that a 9-year estimate interval is optimal; however, Gonedes (1973) suggests that the optimal interval is 7 years. Alexander and Chervang (1980), using mean absolute deviation as a measure of stability, indicate that 4-6-year time intervals produced optimal betas. Reilly and Wright (1988) estimate eight beta 
Table 2 Sample creation

Observations in sample

Entire CRSP universe

$59,171,687$

Beta estimation

Beta estimations

Summarized beta estimations

Average beta estimates per observation

Filtering results

Keep observations with share price greater than $\$ 5.00$

Keep observations with share code equal to 10 or 11

Keep observations with 27 betas
$1,092,009,210$

$54,234,254$

20.14

$44,987,610$

$32,169,860$

$13,588,156$

In this table, we report the evolution our sample takes through each step in the process. Our sample creation process has three main steps. First, we identify the universe of possible securities, which in our case in all of CRSP from 1985 to 2013. Second, we estimate our betas using that universe and summarize all the betas estimates so that each observation contains the summary statistics of the various calculated betas. Third, we filter the sample using common criteria from the literature

coefficients for over 1000 stocks over the period of 1970-1984. Their results indicate that the index selection does not affect estimated betas, but the interval effect is the primary cause for the difference in published betas. They do not posit an optimal time interval. Agrrawal and Clark (2007) analyze the time-dependent factors that impact betas in the Exchange Traded Funds (ETF) markets. Their results based on the betas for the 38 most active ETFs in the US equity markets suggest that betas are highly dependent on the choice of time interval used in their calculation and they recommend using a time interval consistent with the investor's time horizon. Our paper extends Reilly and Wright (1988) results by examining a larger sample of stocks, over 5000 stocks, over a broader period 1985-2013 using three indices. Our results confirm their conclusion that index selection does matter and confirms the time interval effect. Additionally, we examine the relation between beta dispersion and excess returns by calculating approximately 1.1 billion rolling betas based on daily, weekly, and monthly returns.

A number of studies examine the relation between market volatility and stock returns. Giot (2005) shows an asymmetric negative relation between contemporaneous changes in implied volatility and the underlying values of the S\&P 500 and the Nasdaq 100. They show that negative returns for the index are associated with much greater relative movements in the Volitility Index (VIX) than positive returns. Similarly, Ang et al. (2006) find that higher aggregate volatility, based on the VIX, and higher idiosyncratic risk are related to lower average returns. Baker et al. (2011) examine the anomaly of high-beta and high-volatility stocks underperforming low-beta and lowvolatility stocks. They posit a behavioral explanation of this phenomenon. Irrational (or individual) investors tend to prefer lottery behavior and be overconfident which leads to demand for higher-volatility that is not justified by fundamentals, while rational or institutional investors' mandate to beat a fixed benchmark discourages investing in high-alpha, low-beta stocks and low-alpha, high-beta stocks. Baker et al. (2011) examine the relation between beta, volatility, and stock returns, while we examine the relations between beta, beta dispersion, and stock returns. We inspect stock performance by creating low-beta, lowbeta dispersion; low-beta, high-beta dispersion; high-beta, low-beta dispersion; and high-beta, high-beta dispersion portfolios. We anticipate that stocks with greater dispersion in their betas will have potentially larger returns than stocks with more stable betas.

\section{Methodology and data}

We use CRSP data for the period 1985-2013 to estimate over 1.0 billion betas for over 5000 firms using three different time intervals and three market proxies. We estimate 27 different variations of beta using daily, weekly, and monthly returns combined with three different market proxies, the Standard and Poor's 500 index, the CRSP equally weighted index, and the CRSP value-weighted index. For each time interval, we estimate betas for three different time windows. For daily, we use 63, 126, and 252 days; for weekly, we use 52, 104, and 156 weeks; and for monthly, we use 36, 48, and 60 months; thus 27 $(3 \times 3 \times 3)$ variations of estimating beta. Following previous literature, we omit stocks with share prices under $\$ 5$ (Hvidkjaer 2008) and only keep securities with CRSP share codes 10 and 11 in our sample (Chordia and Swaminathan, 2000). See Table 2 for detailed description of sample creation. 


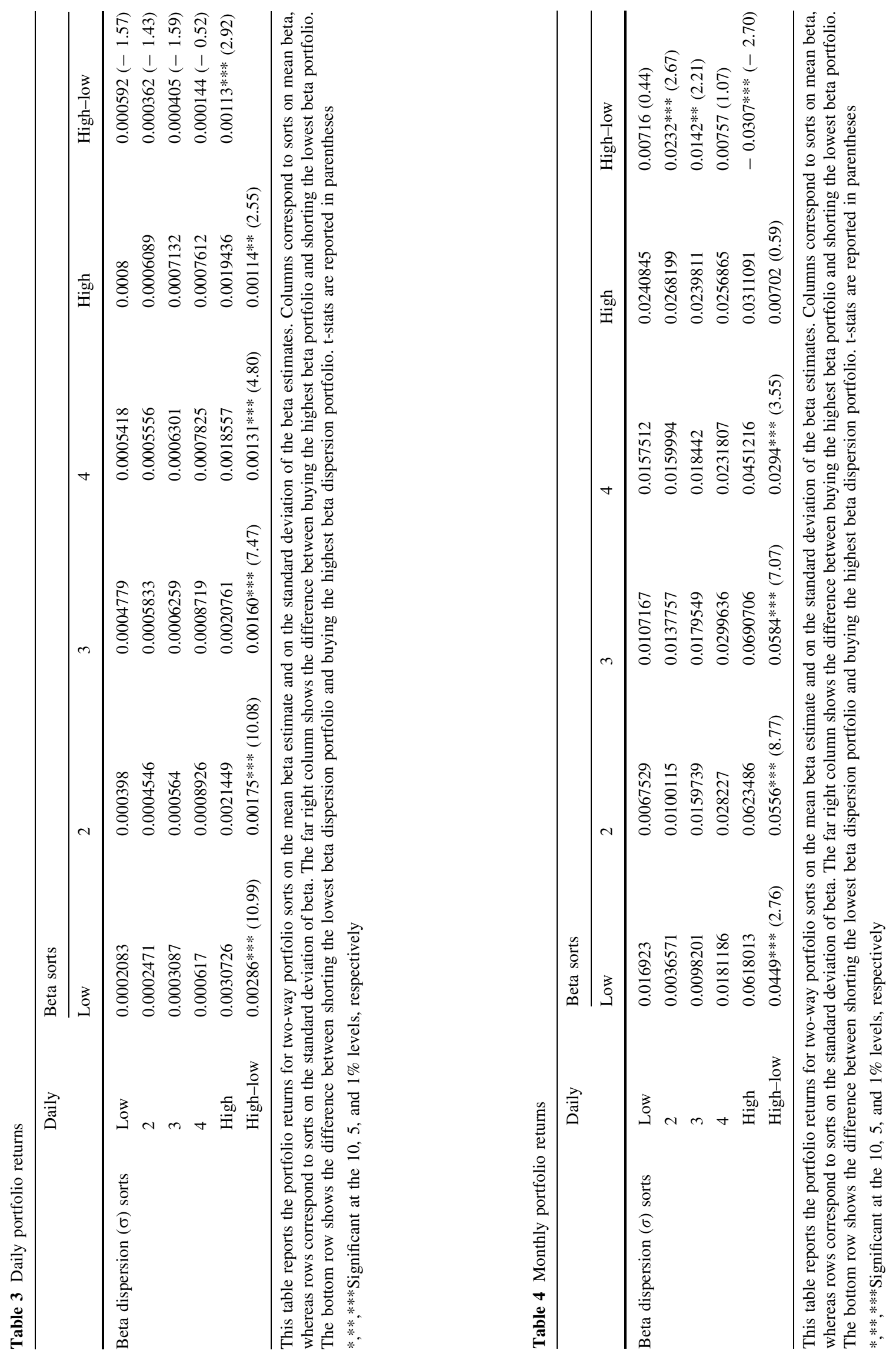


Next, we calculate the standard deviations of the various beta estimates $\left(\sigma_{\beta}\right)$ to analyze the dispersion of beta estimates by method. We hypothesize that stocks whose betas vary more exhibit characteristics of a riskier stock and thus are more likely to have excess returns. We examine the relation between beta dispersion and stock returns by constructing rolling portfolios based on beta dispersion and beta-level quintiles. We calculate two sets of rolling portfolios, one that is rebalanced daily with the other rebalanced monthly.

\section{Results}

Tables 3 and 4 report the returns for two-way portfolio sorts rebalanced daily and monthly. Portfolios are constructed based on the beta-level quintiles and beta dispersion quintiles. Additional portfolios are created by shorting the stocks in the lowest quintile while simultaneously buying the stocks in the highest quintile (long-short) with the results of daily portfolio rebalancing in Table 3 and monthly portfolio rebalancing in Table 4 . The difference between the high dispersion quintiles and the low dispersion quintiles shows highly significant excess returns. The daily portfolio results indicate that the returns of portfolios containing stocks with highly dispersed betas are 15 times greater than the portfolios with low beta dispersion. This result indicates that a single beta may not be capturing all of the "true or relevant" risk, thus allowing for potential excess returns.

Another interesting observation is that the long-short portfolio returns are largest for firms with the lowest betas, 0.00286 in the lowest beta quintile versus 0.00114 in the highest beta quintile, indicating that abnormally low betas may be less accurate estimates of risk of stock returns than typical beta estimates. Results reported in Table 4 for twoway portfolio sorts rebalanced monthly are consistent with daily rebalanced portfolio results, although, statistical significance is lost between the difference of the high-beta dispersion and low-beta dispersion portfolios for high-beta stocks. Based on these results, we conclude that betas of stocks with highly dispersed beta estimates may not be adequately capturing the "true" riskiness of the stocks, especially for firms with below-average betas. Although we only test the statistical difference between highest dispersion quintile and the lowest dispersion quintile, we observe that the high dispersion quintile generates much higher returns than all the other quintiles strengthening our hypothesis that portfolios of stocks with highly dispersed betas will generate higher returns.

\section{Conclusion}

Our analysis of beta dispersion suggests two major conclusions. First, using a much larger sample, we confirm Reilly and Wright's (1988) interval effect by showing betas produced using daily, weekly, and monthly returns differs considerably. Second, we examine a novel measure of return volatility, beta dispersion, measured as the standard deviation of a firm's 27 beta estimates. Portfolio analysis indicates that portfolios constructed of stocks with highly dispersed betas outperform portfolios with low dispersion betas. In daily (monthly) rebalanced portfolios, the highest beta dispersion portfolio has a daily return 15 (3.7) times greater than the portfolio with the lowest dispersion. These results open the door to many interesting questions about how beta dispersion may be priced into security returns, what is the underlying theoretical causes for the differences in performance for each beta dispersion quintile, and whether a trading strategy could be developed that could capture the higher returns generated by stocks with high beta dispersion.

\section{References}

Agrrawal, Pankaj, and John Clark. 2007. ETF betas: A study of their estimation sensitivity to varying time intervals. Institutional Investor Journals, Exchange-Traded Funds \& Indexing Innovations 41: 96-103.

Alexander, Gordon J., and Norman L. Chervang. 1980. On the estimation and stability of beta. The Journal of Finance and Quantitative Analysis 15 (1): 123-137.

Ang, Andrew, Robert J. Hodrick, Yuhang Xing, and Xiaoyan Zhang. 2006. The cross-section of volatility and expected returns. The Journal of Finance XLI (1): 259-299.

Baesel, Jerome B. 1974. On the assessment of risk: Some further considerations. Journal of Finance 29: 1491-1494.

Baker, Malcolm, Brendan Bradley, and Jeffrey Wurgler. 2011. Benchmarks as limits to arbitrage: Understanding the lowvolatility anomaly. Financial Analysts Journal 67 (1): 40-54.

Brenner, Menachem, and Seymour Smidt. 1977. A simple model of non-stationarity of systematic risk. The Journal of Finance 32 (4): 1081-1092.

Chordia, Taron, and Bhaskaran Swaminathan. 2000. Trading volume and cross-autocorrelations in stock returns. The Journal of Finance LV (2): 913-935.

Giot, Pierre. 2005. Relationships between implies volatility indexes and stock index returns. The Journal of Portfolio Management 31 (3): $92-100$.

Gonedes, Nicholas J. 1973. Evidence on the information content of accounting numbers: Accounting-based and market-based estimates of systematic risk. Journal of Financial and Quantitative Analysis 8: 1123-1128.

Hvidkjaer, Soeren. 2008. Small trades and the cross-section of stock returns. Review of Financial Studies 21 (3): 1123-1151.

Reilly, Frank K., and David J. Wright. 1988. A comparison of published betas. The Journal of Portfolio Management 14 (3): 64-69. 
Sharpe, Wiliam F. 1963. A simplifed model for portfolio analysis. Management Science 9 (2): 77-293.

Kyre Dane Lahtinen is an Assistant Professor of Finance in the Department of Economics and Finance at The University of South Alabama in Mobile, Alabama. His research focuses primarily on behavioral finance and empirical asset pricing.

Chris M. Lawrey is an Assistant Professor of Finance in the Department of Economics and Finance at The University of South
Alabama in Mobile, Alabama. His research focuses primarily on working capital topics such as firm liquidity, trade credit, and inventory.

Kenneth J. Hunsader is a Professor of Finance and Chair of the Department of Economics and Finance at The University of South Alabama in Mobile, Alabama. His research focuses primarily on mergers and acquisitions, energy markets, regulation, and event studies. 